\title{
ZnSe Light Emitting Diode Quantum Efficiency and Emission Characterization
}

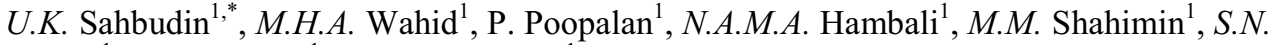 \\ Ariffin $^{1}$, N.N.A. Saidi ${ }^{1}$, and M.M. Ramli ${ }^{1}$. \\ ${ }^{1}$ Semiconductor Photonics \& Integrated Lightwave Systems (SPILS), School of Microelectronic \\ Engineering, Universiti Malaysia Perlis, Pauh Putra Main Campus, Malaysia.
}

\begin{abstract}
ZnSe}$ has demonstrated as a potential candidate in realizing advance LED in some appications for current and future works that utilize a cheaper preparation technique. Blue and white LEDs have been shown to spread across compound semiconductors. This II-VI compound semiconductor with a direct and wide band gap is used in the study which focused on a preparation and its characterization. The device is developed using a circular chip of $\mathrm{ZnSe}$ but only part of the active region is designed to allow shorter computation time. Analyses of the proposed LED are performed in an environment that allows optical transition and nonradiative recombination mechanisms. Voltage variation from $0 \mathrm{~V}$ to $1.5 \mathrm{~V}$ is maintained throughout the observation. The curent-voltage plot shows the p-n junction or diode behavior with central emissive layer. The two dimensions surface emission rate obtained indicates that voltage increment causes the emission concentration to become higher near the central pcontact. The LED efficiency is assessed in terms of internal quantum efficiency and emitting rate.
\end{abstract}

\section{Introduction}

Light emitting diode, LED has attracted many researchers due to its low energy consumption and long lasting operation. It has created trillion dollars business turn over in the last decade for the demand in houses, offices and vehicles. Solid state lightings, SSLs have attracted huge attention especially from wide band gap semiconductors based emitting devices. Many research works are focused in light emitting diodes, LEDs with high efficiency, high colour, low voltage and low temperature.

Researchers have been studying the physics of II-VI and III-V compound materials such as gallium nitride $(\mathrm{GaN})$ and zinc oxide $(\mathrm{ZnO})$ ever since the $1960 \mathrm{~s}$ in order to develop optical devices with shorter wavelengths [1]. Zinc selenide, $\mathrm{ZnSe}$ is one of the II-VI compound semiconductors with wide band gap and also in the category of direct band gap. The $\mathrm{ZnSe}$ has wide range of visible spectrum and often used in electroluminescent display and window layers in solar cells [2]. During the 1990's, ZnSe LED studied in [3] is fabricated by applying p-type layer on the $n-Z n S e$. The $p-n$ junction diode is formed by

\footnotetext{
* Corresponding author: ummikhaireah@gmail.com
} 
introducing lithium, Li to the compensated layers by thermal diffusion. Blue light emission is seen when the structure is in forward bias at room temperature.

Methods in fabricating and producing zinc selenide LEDs have been researched and analyzed to date. Layers of $\mathrm{ZnSe}$ can be formed in ways such as growing them by metalorganic vapour phase epitaxy (MOVPE), physical or chemical vapour deposition and conventional molecular beam epitaxy (MBE). The fabrication of LED is done using conventional photolithographic techniques with device isolation being achieved by wet chemical etching, patterning by vacuum evaporation and lift-off.

The study presented in this paper is devoted to preparation and characterization of $\mathrm{ZnSe}$ LED using a simulated approach, and its comparison with fabricated works. The efficiency of LED and its' relation to LED droop is also discussed. The study is accomplished using COMSOL Multiphysics semiconductor software package.

\section{LED principle operation}

Light emitting diode (LED) is a p-n junction diode made up of layers of semiconductor grown on a thin wafer of crystalline material. The p-type and n-type semiconductor consist of an excess amount of free conducting holes and conduction electrons respectively. Once forward bias, $\mathrm{V}$ is applied, voltage drops across depletion region which allows electron and holes to diffuse across the junction. Electrons from n-side diffuse into p-side whereas holes from $p$-side diffuse into n-side. Electron-hole pair (EHP) recombination occurs in the depletion region which results in spontaneous emission of photon as can be seen in Fig. 1. When all free electrons and holes recombined, enough light will be emitted through the device.

A variation of light colour with specific wavelength can be produced using different kind of semiconductors. As EHP recombination occurs, each photon is emitted at different amount of energy according to the material's bandgap. Therefore, different light colour can be produced. For instance, gallium nitride $(\mathrm{GaN})$ and gallium phosphide produce green LEDs, aluminium gallium indium phosphide (AlGaInP) alloys for red, orange and yellow LEDs while silicon carbide $(\mathrm{SiC})$ and zinc selenide $(\mathrm{ZnSe})$ produce blue LEDs. Slight changes in the composition of these materials govern the colour of the emitted light. White LED can be produced by combining blue, red and green.

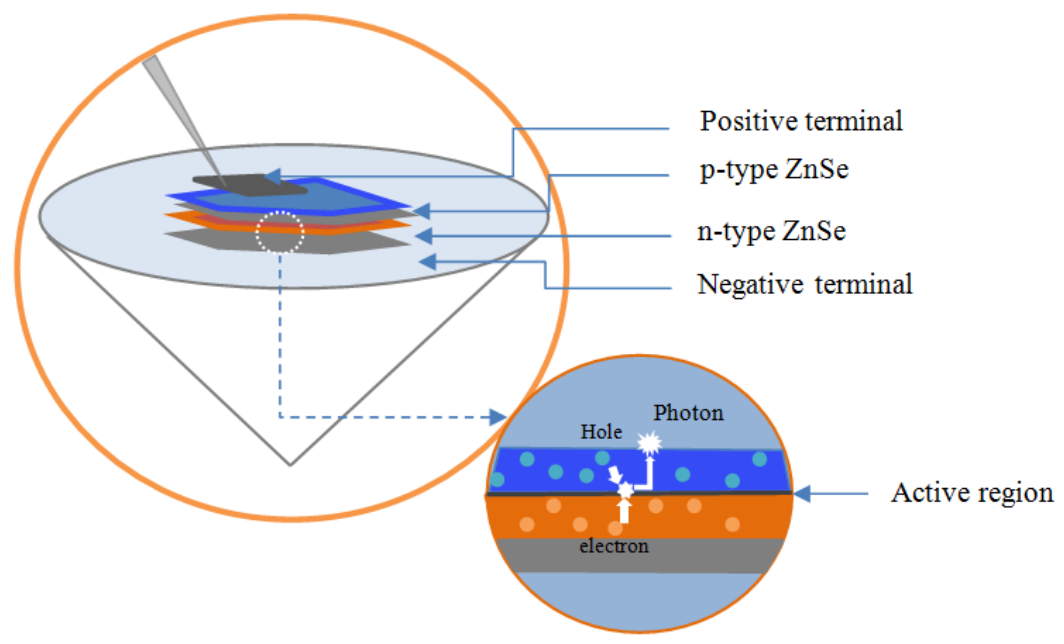

Fig. 1. Illustration of conversion process in LED chip. 


\section{Methodology}

In this work, zinc selenide light emitting diode is evaluated and structurally designed to be cylindrically symmetrical to shorten the required computation time in the three dimension visualization. The design undergoes two different studies or experiments with different choosen parameters. First study is on semiconductor initialization that focuses on mesh and doping whereas the second study analyses the effect of applying voltage to the LED. Both studies investigate the relationship between current and voltage, the emission rate as well as the efficiency of LED in relation to bias voltage and current.

\subsection{LED structure}

A cross section of the active region formed by the $\mathrm{p}$ - and n-type doping is designed as shown in Fig. 2. The whole model is designed in two dimension axisymmetric module under semiconductor physics. The ZnSe layer is $30 \mu \mathrm{m}$ and $10 \mu \mathrm{m}$ in terms of width and height, accordingly. Doping profile of $\mathrm{ZnSe}$ is set using the analytic doping feature where the impurity type and concentration is specified. The top layer $2.5 \mu \mathrm{m}$ of the structure is $\mathrm{p}$ doped while the bottom $7.5 \mu \mathrm{m}$ is $\mathrm{n}$-doped. Part of the structure is etched to the $\mathrm{n}$-type layer and n-contact is deposited on the n-type layer. Then, a $2.5 \mu \mathrm{m}$ width p-type contact is deposited on top surface of ZnSe. Boundary for metal contact is added at both p- and ncontacts to allow electric potential to flow at the terminal.

Two additional features are included in this LED simulation. In order to allow the calculation for electroluminescence of the LED, an optical transition feature is added to the domain. Optical transition is generally, a process where an atom changes from one state of energy to another and thus, emits an electromagnetic radiation. This feature aids in realizing the radiative recombination and spontaneous emission that occurs in the semiconductor. The choosen transition model is the direct band gap which means two-band model is used to represent a direct band gap semiconductor. The spontaneous emission of photons as a result of recombination is computed and analysed.

In terms of EHP recombination, there are radiative recombination that results in photon emission and nonradiative recombination that releases energy in other forms instead of light. Auger effect occurs as EHP recombines and the released energy is passed to another electron in the conduction band which would then dissipates as heat which causes it to be listed as part of nonradiative recombinations [4]. Auger recombination is adopted in order to include nonradiative recombination mechanism and to infer the performance of LED.

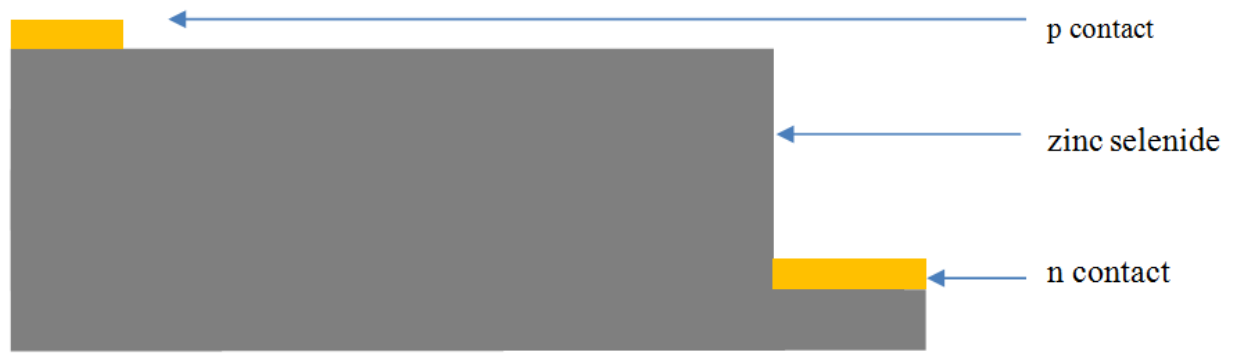

Fig. 2. Cross section of the active region of ZnSe LED.

\section{Results and discussion}

The results obtained from the first study are the $2 \mathrm{D}$ meshes and doping gradient. The default mesh, with the size set to fine, is shown in Fig. 3.This simulation focuses in 
resolving the p-n junction region, thus, no special scales are specified for the mesh. Mesh is used to represent the solution field to the physics being solved which in this case is the doping gradient. Fig. 4 shows the solution for the first study where the mesh is refined around the doping profile. The mesh is also tighter at the area with $\mathrm{p}$ and $\mathrm{n}$ electrical contact in order to resolve the high current density and electric field effect. Fig. 5 shows the distribution of dopant throughout the structure. Red area at the bottom indicates the uniform $\mathrm{n}$-doped region and the p-doped surface layer is displayed in blue. The colour that changes steadily from red to blue reflects the doping transition from $\mathrm{n}$ to $\mathrm{p}$-type region in order to create a region where light emission can occur.

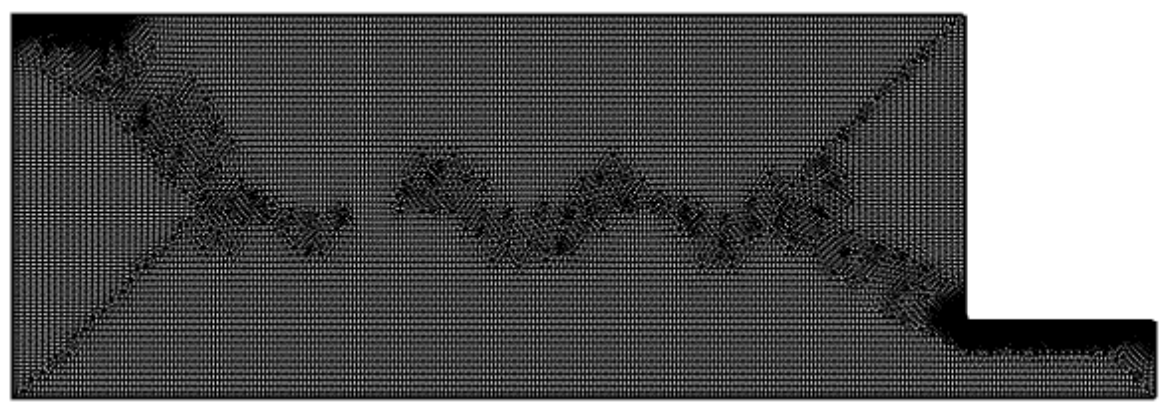

Fig. 3. Induced mesh set to Fine.

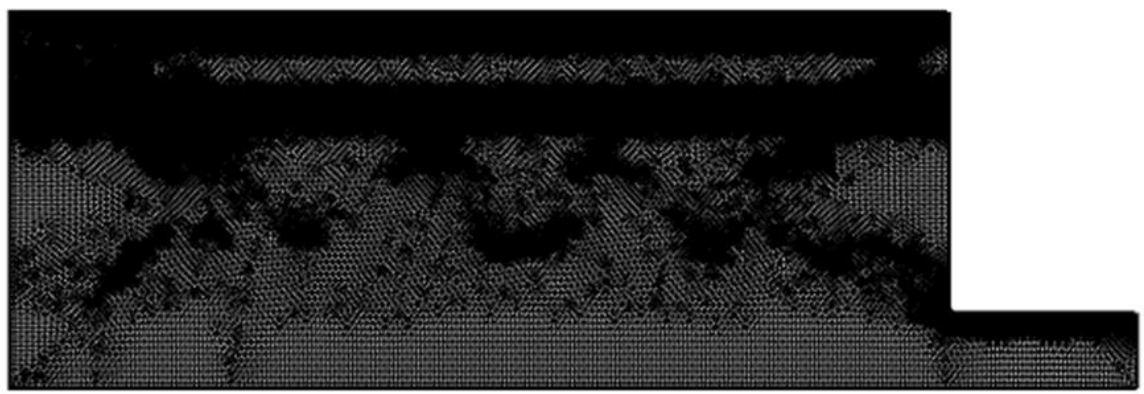

Fig. 4. Refined mesh output.

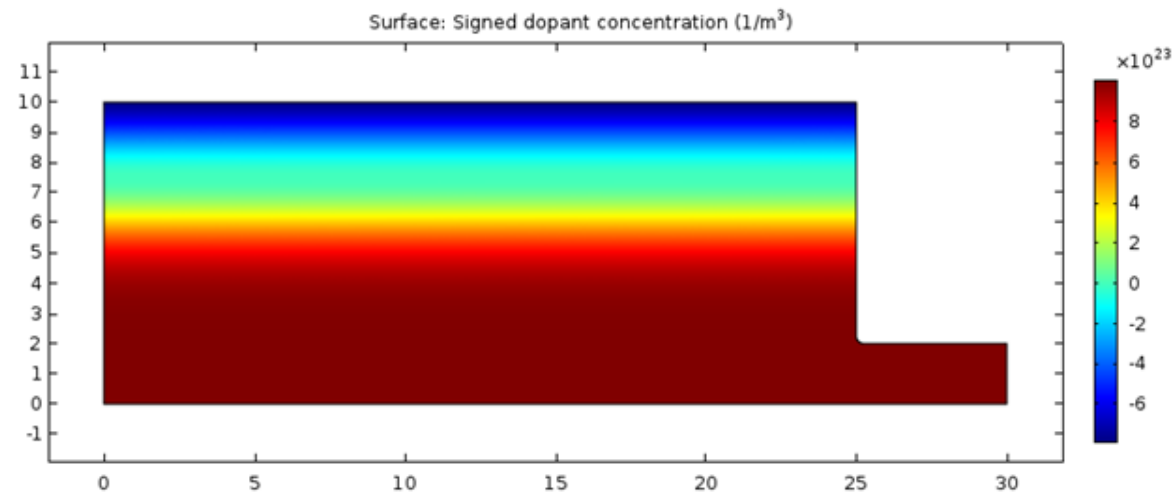

Fig. 5. Two dimension surface graph of signed dopant concentration.

Fig. 6 shows the current voltage curve of the simulated LED from the second study. The graph shows that the LED is a diode with a turn-on voltage of $1.4 \mathrm{~V}$. This clearly shows 
that the ZnSe LED displays the expected diode behaviour as obtained in other reported researches $[5,6]$.

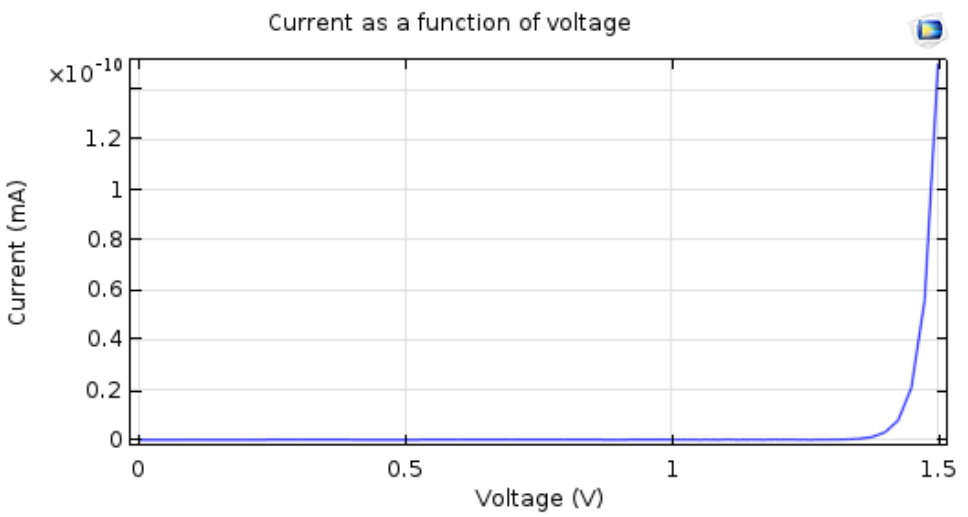

Fig. 6. Current voltage curve.

The following two figures represent the result of the two dimension emission rate across the LED structure. Observing Fig. 7 and Fig. 8 that show the emission rate at $1.0 \mathrm{~V}$ and 1.5 $\mathrm{V}$, respectively, proves that varying the voltage affects the rate of emission. The emission can be seen to spread radially at voltage just above the turn-on voltage. However, voltage increment causes the emission to become intense at the center of the device.

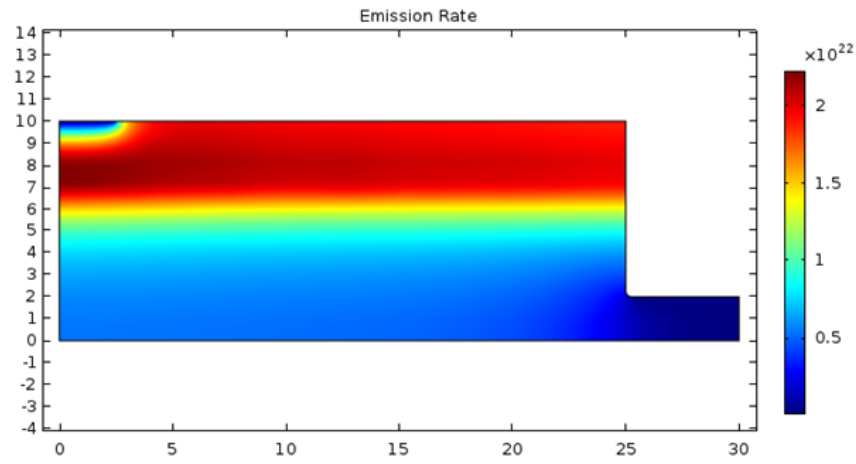

Fig. 7. Two dimension surface graph of emission rtae at voltage bias of $1.2 \mathrm{~V}$.

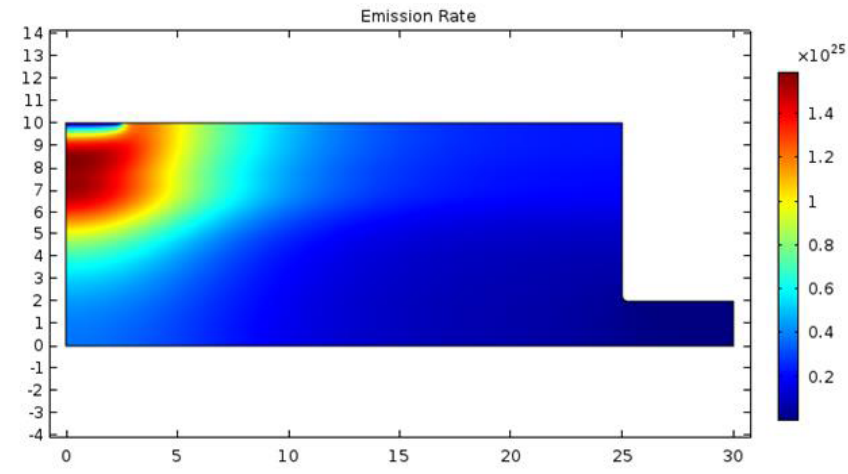

Fig. 8. Two dimension surface graph of emission rtae at voltage bias of $1.5 \mathrm{~V}$. 
Fig. 9 shows one-dimensional plot of the total emission rate against current. The rate of emission increases linearly at the start but it then curves sub-linearly after a few mA. The sub-linear curve is a characteristic of LED droop. As mentioned earlier, LED droop is a phenomenon that occurs in LEDs and the Auger effect is one of the reasons behind the LEDs' efficiency droop. Research done by S. Verma in [4] mentions that Auger effects gives an impact on the quantum efficiency, luminous power and electroluminescence intensity which explains the obtained results..

The decrease in efficiency of the LED can be considered by referring to Fig. 10 that shows the internal quantum efficiency (IQE) as a function of current. IQE is the ratio of emitted photon to the carriers in the active region. The IQE value starts off low but as current flows, there is an abrupt peak in IQE. At higher injection current, the IQE is reduced, thus, showing evidence of LED droop.

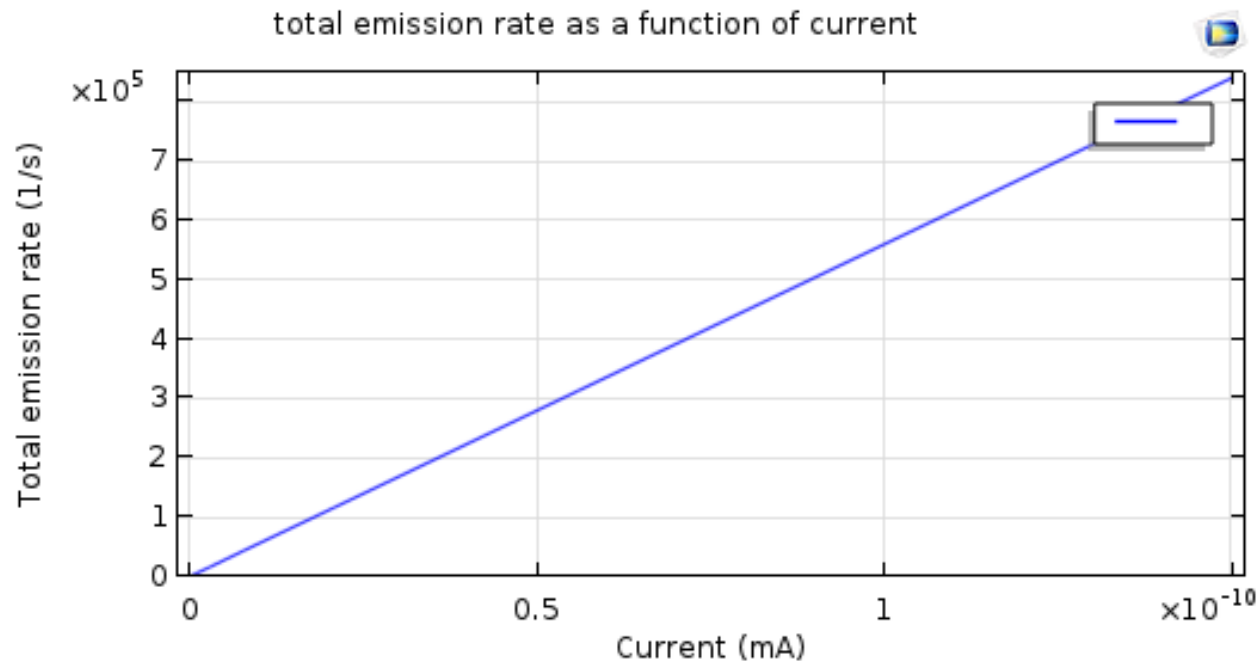

Fig. 9. Total emission rate against current.

Internal Quantum Efficiency as a function of current

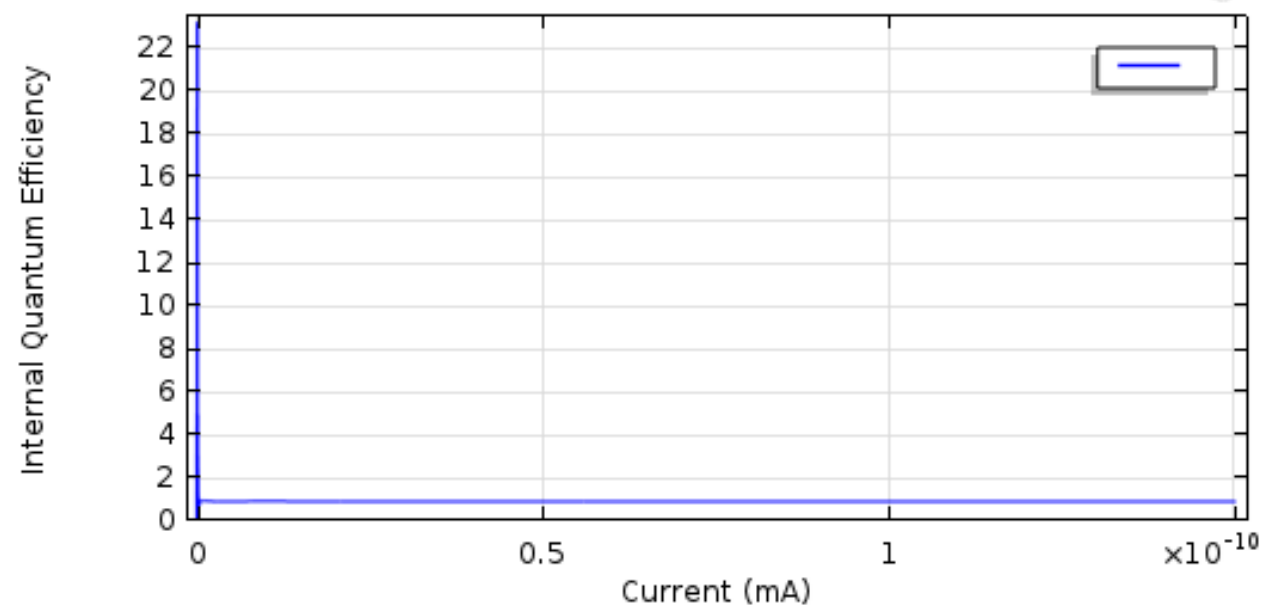

Fig. 10. Internal quantum efficiency against current. 
Fig. 11 shows the cross sectional area of the LED structure. This is the 3D version of the spatial distribution of emission of Fig. 8 with $1.5 \mathrm{~V}$ applied bias. By observing the colour gradients, it can be concluded that the emission becomes concentrated underneath the p-contact surface.

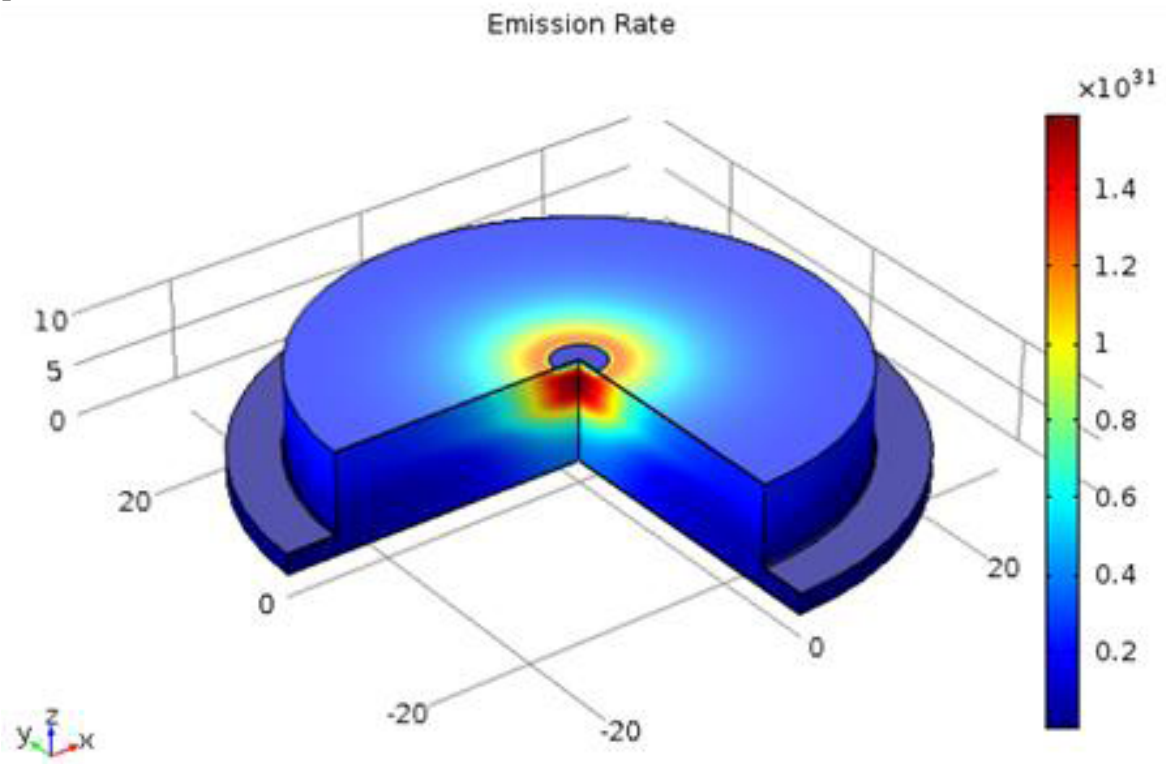

Fig. 11. 3D visualization of LED cross section.

\section{Conclusions}

In this work, a simple ZnSe LED is modeled and analyzed using the semiconductor module in COMSOL. The evaluated LED is made up of $\mathrm{ZnSe}$ layer that is p-doped and $\mathrm{n}$-doped specifically. Metal contacts are deposited on those layers thus, allowing electrical current to flow through the structure. From the two studies on the structure, it is found that the IV curve displayed the expected diode behavior as the fabricated ones. Aside from that, the emission rate and the efficiency of LED is also analyzed. Eventhough it is possible to increase LED brightness by applying larger current, the efficiency of the LED will eventually decrease that end with a droop. Future study of this LED could include varying the doped material and evaluating the performance of the device in different voltages and a different nonradiative recombination mechanism.

\section{References}

1. T. Shirakawa, Mater. Sci. and Engin. B, 91, 470 (2002)

2. S. Muthumari, G. Devi, P. Revathi, R. Vijayalakshmi, C. Sanjeeviraja, Journal of Applied Sciences, 12 (16), 1722 (1992)

3. A.N. Krasnov, Y.N. Purtov, Y.F. Vaksman, V.V. Serdyuk, J. Cryst. Growth, 125 (12), 373 (1992)

4. Y. Namikawa, Sei Technical Review, 72 (2011)

5. V.P. Sirkeli, O. Yilmazoglu, F. Kuppers, H.L. Hartnagel, Semiconductor Science and Technology, 30 (2015) 